\title{
Computational analysis of physico-chemical properties and homology modeling of carbonic anhydrase from Cordyceps militaris
}

\author{
Vivek Dhar Dwivedi · Shikha Arora • \\ Amit Pandey
}

Received: 7 April 2013/Revised: 17 May 2013/Accepted: 9 June 2013/Published online: 18 June 2013

(C) Springer-Verlag Wien 2013

\begin{abstract}
In the present study, the protein sequence of carbonic anhydrase enzyme from Cordyceps militaris (accession no. EGX89555.1) was retrieved from GenPept database and subjected to computation for various physicochemical properties, transmembrane segment prediction, homology modeling, domain identification and structural alignment. Five potential transmembrane segments of various lengths were predicted by transmembrane prediction server. The 3D structure of this protein was determined by homology modeling using $3 \mathrm{~d}$ jigsaw server. A single eukaryotic-type carbonic anhydrase domain belonging to Carb_anhydrases family domain was identified at two positions from 45 to 153 and 232 to 302 residues region. This modeled structure showed $100 \%$ structural similarity with human carbonic anhydrase III (PDB ID: 1z93-A) in PDB database.
\end{abstract}

Keywords Carbonic anhydrase - Cordyceps militaris . Physico-chemical properties - Homology modeling · Domain

\section{Introduction}

Carbonic anhydrase (CA) enzyme was first reported in vertebrate erythrocytes (Brinkman et al. 1932). It forms a family of enzymes that catalyze the rapid interconversion of carbon dioxide and water to bicarbonate and protons (or vice versa), a reversible reaction that occurs rather slowly in the absence of a catalyst (Badger and Price 1994). This

V. D. Dwivedi $(\bowtie) \cdot$ S. Arora $\cdot$ A. Pandey

Forest Pathology Division, Forest Research Institute, Dehradun, India

e-mail: vivek_bioinformatics@yahoo.com enzyme plays a central role in facilitating the diffusion of carbon dioxide in photosynthesis, but it is also essential in areas such as ion balance, respiration and an important role in biosynthesis or detoxification pathways that use $\mathrm{HCO}_{3}$ as a co-factor or as a co-substrate, such as fatty acid or arginine biosynthesis, the cAMP pathway and cyanate degradation (Aguilera et al. 2005; Anderson et al. 1990; Bahn and Mühlschlegel 2006). The Cordyceps militaris occurs throughout much of the Northern Hemisphere as a pathogen of lepidopteran insect pupae (Sung et al. 2007). C. militaris is readily characterized by the sexual fruiting bodies forming on mycosed pupae, the structures giving the fungus its common name of 'pupa grass' in China. The C. militaris is best known as traditional Chinese medicines (Paterson 2008). Extracts from both mycelium and fruiting bodies of $C$. sinensis, C. militaris and other Cordyceps species showed significant anticancer activities by various mechanisms such as, modulating immune system and inducing cell apoptosis (Khan et al. 2010). Over expression of carbonic anhydrase III reduces steady-state levels of intracellular reactive oxygen species, increases proliferation rate and protects cells from hydrogen peroxideinduced apoptosis (Raisanen et al. 1999). In light of above the homology modeling of carbonic anhydrase in C. militaris and its analogy with carbonic anhydrase report in human system becomes very important in understanding the much acclaimed role of $C$. militaris as antiaging, anticancerous and immunomodulating agent. Considering the above facts, the study of amino acid sequence of carbonic anhydrase from $C$. militaris is a very interesting task. In this communication, we performed the in-silco analysis of $C$. militaris carbonic anhydrase to find out the physical and chemical properties like molecular weight, theoretical pI, atomic composition, estimated half-life, instability index, aliphatic index and grand average of hydropathicity 
on the basis of their amino acid sequence. Transmembrane segment prediction, domain identification and the automatic comparative modeling of carbonic anhydrase from C. militaris were also performed.

\section{Materials and methods}

The protein sequence of carbonic anhydrase enzyme from C. militaris (accession no. EGX89555.1) was searched and retrieved from GenPept database available at NCBI website (http://www.ncbi.nlm.nih.gov/protein). Computation of various physical and chemical properties including the molecular weight, theoretical pI, atomic composition,

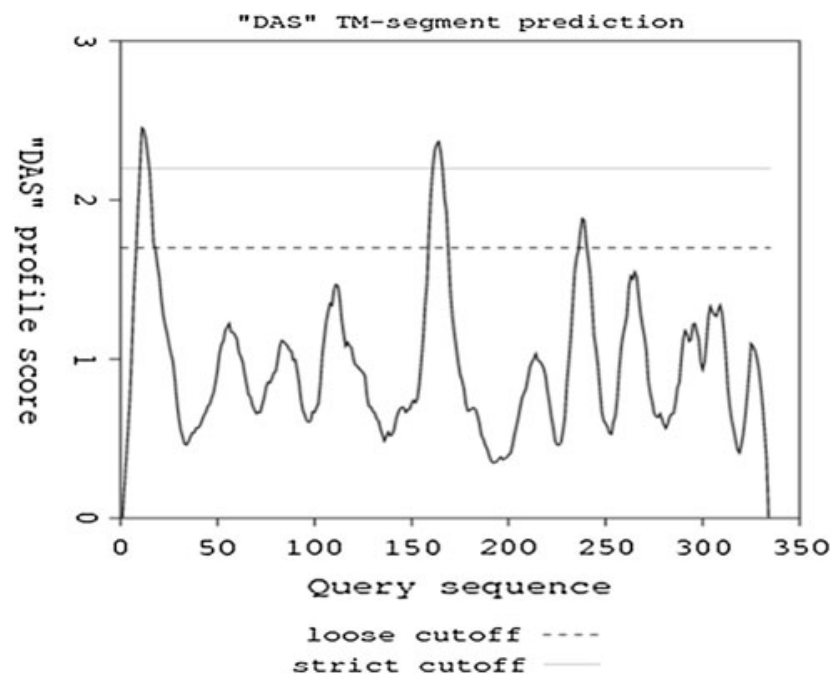

Fig. 1 Transmembrane segment prediction by DAS server estimated half-life, instability index, aliphatic index and grand average of hydropathicity was calculated using ProtParam tool (http://web.expasy.org/protparam/) (Gasteiger et al. 2005). Transmembrane segment prediction was performed using "DAS"-Transmembrane Prediction server (http://www.sbc.su.se/ miklos/DAS/) (Cserzo et al. 1997). Homology modeling was performed using 3D jigsaw server (http://bmm.cancerresearchuk.org/ 3djigsaw/) (Bates et al. 2001) and modeled structure was visualized and annotated under Rasmol program and SAS (http://www. ebi.ac.uk/thornton-srv/databases/sas/). Domain was identified using Pfam database search program (http://pfam.

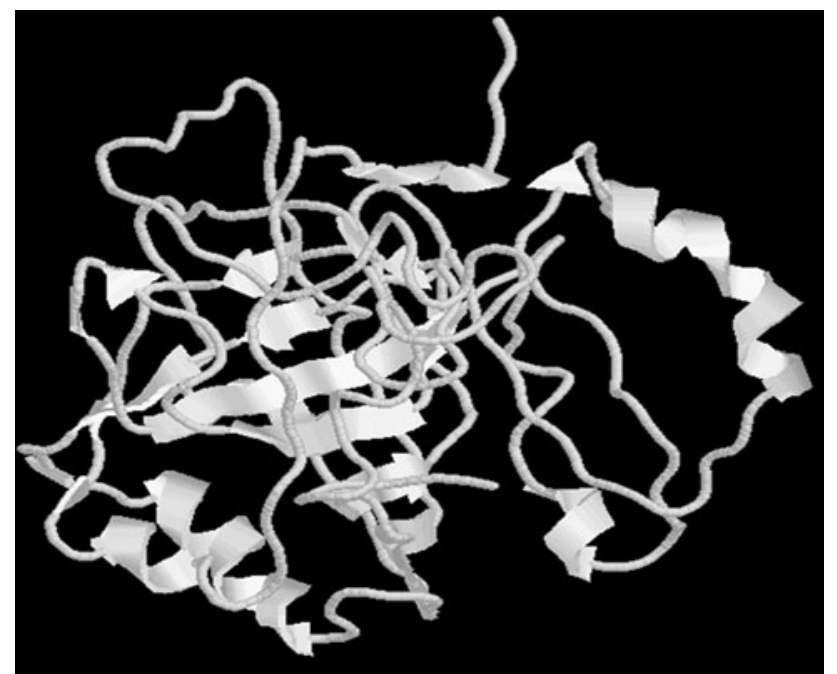

Fig. 2 3D structure of carbonic anhydrase from Cordyceps militaris fungus modeled by $3 \mathrm{~d}$ jigsaw server and visualized under Rasmol program

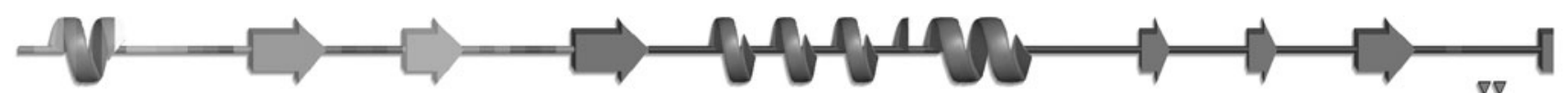

MLLRKVVGMSLLLPAVQVSASCGYGT ILHPRAENGTLEVKDFGYFGSKGPTWWALDPTANALCASGNSQS P INLVKGSQS IVPAAELKLA I PDMPDGAT

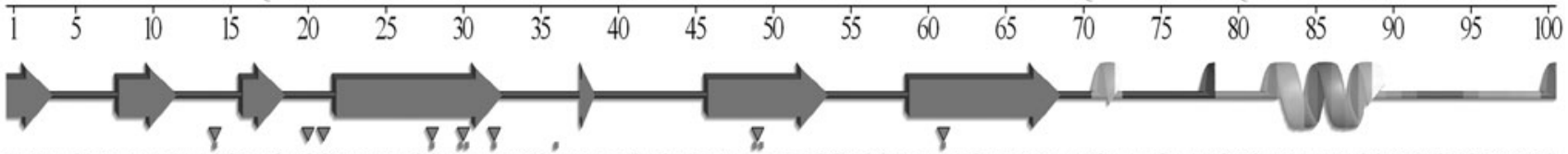

FENLGTTVEVVAAGGMMSGGTAYTLQQFHFHLPSEHLDNGASMAMEMHMWQGAGGEVAVVGVFVDIADGAAAGAAPQDGAVAAEAQSRRRSPSLRTRS

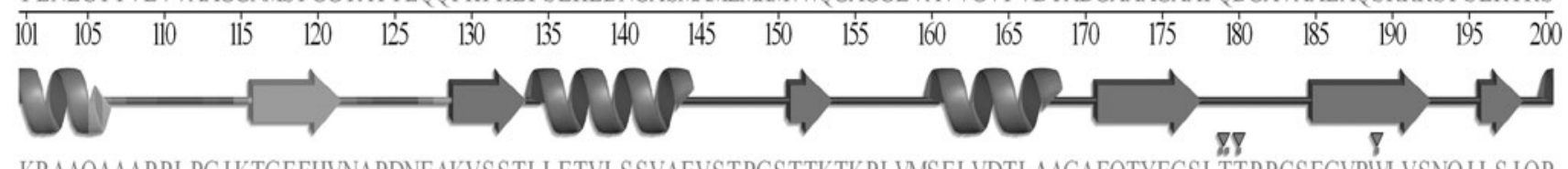

KRAAQAAAPPLPGIKTGFFHVNAPDNEAKVSSTLLETVLSSVAEVSTPGSTTKTKPLVMSELVDTLAAGAFQTYEGSLTTPPCSEGVRWLVSNQILS IQP

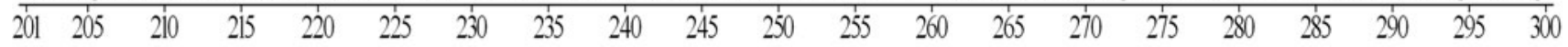

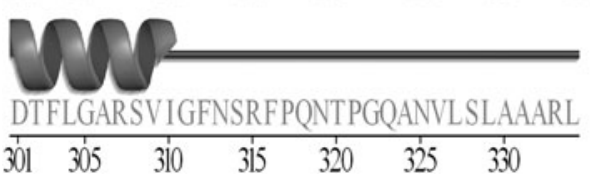

Fig. 3 Model analyzed under SAS server available at EBI 


\begin{tabular}{|c|c|c|c|c|c|c|c|c|c|c|c|}
\hline $0:$ & & $\mathrm{z}$ & $\mathrm{msd}$ & $1 a 1 i$ & nres & zid & PDB & Descriptic & & & \\
\hline 1: & $1 \mathrm{z} 93-\mathrm{A}$ & 99.9 & 0.5 & 263 & 263 & 100 & $\underline{\text { PDB }}$ & MOLECULE: & CARBONIC & ANHYDRASE & III; \\
\hline$\underline{2}:$ & $1297-\mathrm{A}$ & 5.5 & 0.0 & 263 & 263 & 100 & $\underline{\mathrm{PDB}}$ & MOLECULE: & CARBONIC & ANHYDRASE & III; \\
\hline$\underline{3}:$ & $1 \pm 1 j-A$ & 4.5 & 0.6 & 257 & 259 & 91 & $\underline{\mathrm{PDB}}$ & MOLECULE: & CARBONIC & ANHYDRASE & III; \\
\hline 4 : & $2 h f y-A$ & 4.3 & .5 & 257 & 259 & 99 & $\underline{\text { PDB }}$ & MOLECULE : & CARBONIC & ANHYDRASE & $3 ;$ \\
\hline$\underline{5}:$ & $2 \mathrm{hfw}-\mathrm{A}$ & 44.2 & 0.5 & 257 & 257 & 99 & $\underline{\text { PDB }}$ & MOLECULE: & CARBONIC & NHYDRASE & 3; \\
\hline$\underline{6}:$ & $2 h f x-A$ & 44.1 & 0.6 & 257 & 259 & 99 & $\underline{\mathrm{PDB}}$ & MOLECULE : & CARBONIC & INHYDRASE & 3 ; \\
\hline$\underline{7}:$ & $3 \mathrm{daz}-\mathrm{A}$ & 41.7 & 1.0 & 257 & 257 & 59 & $\underline{\text { PDB }}$ & MOLECULE: & CARBONIC & RRASE & 2; \\
\hline ㅇ: & $3 \mathrm{dc} 9-\mathrm{A}$ & 41.7 & 1.0 & 257 & 257 & 59 & $\underline{\mathrm{PDB}}$ & MOLECULE: & CARBONIC & ANHYDRASE & 2: \\
\hline$\underline{9}:$ & 1 th9-A & 41.7 & 1.0 & 257 & 258 & 59 & $\underline{\text { PDB }}$ & MOLECULE: & CARBONIC & YDRASE & II; \\
\hline 10: & 1 thk-A & 41.7 & 1.0 & 257 & 258 & 59 & $\underline{\mathrm{PDB}}$ & MOLECULE: & CARBONIC & ANHYDRASE & II; \\
\hline 11: & $119 \mathrm{~m}-\mathrm{A}$ & 41.7 & 1.0 & 257 & 258 & 59 & $\underline{\text { PDB }}$ & MOLECULE: & CARBONIC & YDRASE & II; \\
\hline 12: & $3 \mathrm{dbu}-\mathrm{A}$ & 41.7 & 1.0 & 257 & 257 & 59 & $\underline{\mathrm{PDB}}$ & MOLECULE: & CARBONIC & YDRASE & $2 ;$ \\
\hline 13: & 2nwy-A & 41.7 & 1.0 & 257 & 258 & 59 & $\underline{\mathrm{PDB}}$ & MOLECULE: & CARBC & YDRASE & 2; \\
\hline 14: & $2 \mathrm{n} 4 \mathrm{n}-\mathrm{A}$ & 41.7 & 1.0 & 257 & 257 & 59 & $\underline{\mathrm{PDB}}$ & MOLECULE: & CARBC & YDRASE & II ; \\
\hline 15: & $203 f-A$ & 41.6 & 1.0 & 257 & 257 & 59 & $\underline{\mathrm{PDB}}$ & MOLECULE: & CARB & DRASE & $2 ;$ \\
\hline 16: & $2 a b e-A$ & 41.6 & 1.0 & 257 & 257 & 59 & $\underline{\mathrm{PDB}}$ & MOLECULE: & CARBONIC & IDRASE & II; \\
\hline 17: & $1 \mathrm{zsb}-\mathrm{A}$ & 41.6 & 0.9 & 257 & 257 & 59 & $\underline{\mathrm{PDB}}$ & MOLECULE: & CARB & RASE & II; \\
\hline 18: & $1 \times \mathrm{zC}-\mathrm{A}$ & 41.6 & 1.0 & 257 & 258 & 59 & $\underline{\mathrm{PDB}}$ & MOLECULE: & CARBONIC & YDRASE & II ; \\
\hline 19: & $1 \mathrm{n} 4 \mathrm{n}-\mathrm{A}$ & 41.6 & 1.0 & 257 & 25 & 59 & $\underline{\mathrm{PDB}}$ & MOLECULE: & CARBONIC & RASE & II ; \\
\hline 20: & $1 \mathrm{~g} 52-\mathrm{A}$ & 41.6 & 1.0 & 257 & 258 & 59 & $\underline{\text { PDB }}$ & MOLECULE: & CARBONIC & CDRASE & II ; \\
\hline 21: & $1 \mathrm{~g} 46-\mathrm{A}$ & 41.6 & 0.9 & 257 & 258 & 59 & $\underline{\text { PDB }}$ & MOLECULE: & CARB & RASE & II ; \\
\hline 22: & 1 teq $-x$ & 41.6 & 1.0 & 257 & 258 & 59 & $\underline{\mathrm{PDB}}$ & MOLECULE: & CARBONIC & RASE & II; \\
\hline 23: & $1 \mathrm{cni}-\mathrm{A}$ & 41.6 & 1.0 & 257 & 257 & 59 & $\underline{\text { PDB }}$ & MOLECULE: & CARBONIC & RASE & II; \\
\hline 24: & 1 yo2-A & 41.6 & 1.0 & 257 & 258 & 59 & $\underline{\mathrm{PDB}}$ & MOLECULE: & CARBONI & RASE & II; \\
\hline 25: & 1 yo1-A & 41.6 & 1.0 & 257 & 258 & 59 & $\underline{\text { PDB }}$ & MOLECULE: & CARBONIC & RASE & II ; \\
\hline 26: & $2 n \times x-A$ & 41.6 & 1.0 & 257 & 258 & 59 & $\underline{\mathrm{PDB}}$ & MOLECULE: & CARBONIC & RASE & 2; \\
\hline 27: & $1 \mathrm{cnh}-\mathrm{A}$ & 41.6 & 1.0 & 257 & 257 & 59 & $\underline{\text { PDB }}$ & MOLECULE : & CARBONIC & RASE & II: \\
\hline 28: & $119 q-A$ & 41.6 & 1.0 & 257 & 258 & 59 & $\underline{\mathrm{PDB}}$ & MOLECULE: & CARBONIC & ANHYDRASE & II ; \\
\hline 29: & $1 \mathrm{~g} 3 \mathrm{z}-\mathrm{A}$ & 41.6 & 1.0 & 257 & 258 & 59 & PDB & MOLECULE: & CARBONIC & ANHYDRASE & II: \\
\hline
\end{tabular}

Fig. 4 Structure of C. militaris carbonic anhydrase showing $100 \%$ similarity with human carbonic anhydrase III (PDB ID: 1z93-A) in PDB database

sanger.ac.uk/search) (Finn et al. 2010). Structural alignment was performed using Dali server (http://ekhidna. biocenter.helsinki.fi/dali_server/start).

\section{Results and discussion}

The amino acid sequence of carbonic anhydrase enzyme from $C$. militaris fungus was searched and retrieved from GenPept database of NCBI along with the accession number XP_001389200.2. The computation of various physical and chemical properties result revealed that atomic composition consisted of 1,527 carbon, 2,428 hydrogen, 422 nitrogen, 471 oxygen and 12 sulfur atoms and molecular weight was found to be $34.6 \mathrm{kD}$. The theoretical pI was estimated to be 5.87. Half-life, instability index, aliphatic index and grand average of hydropathicity were $20 \mathrm{~h}, 48.6$, 85.93 and 0.092 , respectively. Instability index was $>40$ so this protein is unstable. Five potential transmembrane segments of various lengths were predicted by transmembrane prediction server (Fig. 1). First segment was nine residues long and found from 9 to 17 residues region with 1.7 cutoff value, second segment was four residues long and found from 11 to 14 residues region with 2.2 cutoff value, third segment was ten residues long found from 159 to 168 residues region with 1.7 cutoff value, fourth segment was five residues long found from 161 to 165 residues region with 2.2 cutoff value and fifth segment was four residues long found from 237 to 240 residues region with 1.7 cutoff value. Homology modeling of retrieved amino acid sequence was performed using $3 \mathrm{~d}$ jigsaw server to get the $3 \mathrm{D}$ coordinates. The model structure was visualized and annotated using Rasmol program and SAS server respectively (Figs. 2, 3). Using Rasmol program it was found that the 3D structure of carbonic anhydrase enzyme from $C$. militaris consisted of 9 alpha helices, 18 beta strands and 34 turns, stabilized by 123 hydrogen bonds. The domain identification result showed single eukaryotic-type carbonic anhydrase domain belonging to Carb_anhydrases family domain at two positions from 45 to 153 and 232 to 302 residues regions. Structural alignment of $C$. militaris carbonic anhydrase using Dali server showed $100 \%$ similarity with human carbonic anhydrase III (PDB ID: 1z93-A) in PDB database (Fig. 4). 


\section{Conclusions}

The amino acid sequence-based physico-chemical properties analysis of $C$. militaris carbonic anhydrase revealed that this protein is unstable with $34.6 \mathrm{kD}$ molecular weight and carries no net electrical charge at $5.87 \mathrm{pH}$ value. Homology modeling result showed that structure of this protein consisted of 9 alpha helices, 18 beta strands and 34 turns which were stabilized by 123 hydrogen bonds and single eukaryotic-type carbonic anhydrase domain belonging to Carb_anhydrases family identified at two positions (45-153 and 232-302) using Pfam database. Modeled structure showed $100 \%$ similarity with human carbonic anhydrase III (PDB ID: 1z93-A) in PDB database. The structural similarity of carbonic anhydrase of $C$. militaris with Carbonic anhydrase III of human provides evidence for its role as an antiaging, anticancerous and immunomodulating agent. However, owing to the considerable importance of carbonic anhydrase in C. militaris, more contributions are warranted for the detailed investigation of the functional properties of this enzyme with respect to human carbonic anhydrase III.

Acknowledgments We are thankful to the Head of Forest Pathology Division, Forest Research Institute, Dehradun, India for providing laboratory facilities and encouragement. We are grateful to Dr. Sarad Kumar Mishra, Department of Biotechnology, D.D.U. Gorakhpur University, Gorakhpur, India for his kind support and necessary suggestions whenever needed.

\section{References}

Aguilera J, Van DJP, Winde JH, Pronk JT (2005) Carbonic anhydrase (Nce103p): an essential biosynthetic enzyme for growth of
Saccharomyces cerevisiae at atmospheric carbon dioxide pressure. Biochem J 391:311-316

Anderson PM, Sung YC, Fuchs JA (1990) The cyanase operon and cyanate metabolism. FEMS Microbiol Rev 7:247-252

Badger MR, Price GD (1994) The role of carbonic anhydrase in photosynthesis. Annu Rev Plant Physiol Plant Mol Biol 45: 369-392

Bahn YS, Mühlschlegel FA (2006) $\mathrm{CO}_{2}$ sensing in fungi and beyond. Curr Opin Microbiol 19:572-578

Bates PA, Kelley LA, MacCallum RM, Sternberg MJE (2001) Enhancement of protein modelling by human intervention in applying the automatic programs 3D-JIGSAW and 3D-PSSM. Proteins Struct Funct Genet Suppl 5:39-46

Brinkman R, Margaria R, Meldrum NU, Roughton FJW (1932) The $\mathrm{CO}_{2}$ catalyst present in blood. J Physiol (Lond) 75:3-4

Cserzo M, Wallin E, Simon I, von Heijne G, Elofsson A (1997) Prediction of transmembrane alpha-helices in prokaryotic membrane proteins: the dense alignment surface method. Protein Eng 10:673-676

Finn RD, Mistry J, Tate J, Coggill P, Heger A, Pollington JE, Gavin OL, Gunesekaran P, Ceric G, Forslund K, Holm L, Sonnhammer EL, Eddy SR, Bateman A (2010) Protein family (Pfam) database. Nucleic Acids Res Database Issue 38:D211-D222

Gasteiger E, Hoogland C, Gattiker A, Duvaud S, Wilkins MR, Appel RD, Bairoch A (2005) Protein identification and analysis tools in the ExPASy server. The Proteomics Protocols Handbook. Humana Press, New York, pp 571-607

Khan MA, Tania M, Zhang DZ, Chen HC (2010) Cordyceps Mushroom: A potent anticancer nutraceutical. Open Nutra J 3: 179-183

Paterson RR (2008) A traditional Chinese medicine and another fungal therapeutic biofactory? Phytochemistry 69:1469-1495

Raisanen SR, Lehenkari P, Tasanen M, Rahkila P, Harkonen PL, Vaananen HK (1999) Carbonic anhydrase III protects cells from hydrogen peroxide-induced apoptosis. Faseb J 13:513-522

Sung GH, Hywel-Jones NL, Sung JM, Luangsa-Ard JJ, Shrestha B, Spatafora JW (2007) Phylogenetic classification of Cordyceps and the clavicipitaceous fungi. Stud Mycol 57:5-59 